\title{
Ising Phases of Heisenberg Ladders in a Magnetic Field
}

\author{
Karlo Penc, ${ }^{1}$ Jean-Baptiste Fouet, ${ }^{2}$ Shin Miyahara, ${ }^{3}$ Oleg Tchernyshyov, ${ }^{4}$ and Frédéric Mila ${ }^{5}$ \\ ${ }^{1}$ Research Institute for Theoretical Solid State Physics and Optics, H-1525 Budapest, P.O.B. 49, Hungary \\ ${ }^{2}$ Institut Romand de Recherche Numérique en Physique des Matériaux (IRRMA), CH-1015 Lausanne, Switzerland \\ ${ }^{3}$ Department of Physics and Mathematics, Aoyama Gakuin University, Sagamihara 229-8558, Japan \\ ${ }^{4}$ Department of Physics and Astronomy, Johns Hopkins University, Baltimore, Maryland 21218, USA \\ ${ }^{5}$ Institute of Theoretical Physics, Ecole Polytechnique Fédérale de Lausanne, CH-1015 Lausanne, Switzerland
}

(Received 27 February 2007; revised manuscript received 8 June 2007; published 10 September 2007)

We show that Dzyaloshinskii-Moriya (DM) interactions can substantially modify the phase diagram of spin-1/2 Heisenberg ladders in a magnetic field provided they compete with exchange. For nonfrustrated ladders, they induce a local magnetization along the DM vector that turns the gapless intermediate phase into an Ising phase with broken translational symmetry, while for frustrated ladders, they extend the Ising order of the half-integer plateau to the surrounding gapless phases of the purely Heisenberg case. Implications for experimental ladder and dimer systems are discussed.

DOI: 10.1103/PhysRevLett.99.117201

PACS numbers: 75.10.Jm

Spin ladders [1] have played an important role in the field of strongly correlated systems over the past 15 years. They are among the best studied spin-gap systems, thanks to the numerous experimental realizations in transition metal oxides [2] and organometallic compounds [3]; their intermediate phase in a magnetic field is one of the simplest realizations of a Luttinger liquid [4] and frustrating them with diagonal rungs opens a magnetization plateau at half-saturation [5], a simple example of noninteger magnetization plateaux [6].

All this is true for purely $\mathrm{SU}(2)$ Heisenberg interactions, but in actual systems, anisotropic terms such as the Dzyaloshinskii-Moriya (DM) interaction are often present. In ordered antiferromagnets, they open a small gap in the spin-wave spectrum and may induce a small canting, but otherwise they can be neglected for most purposes. One may thus be tempted to conjecture that, for gapped antiferromagnets, their effect will be negligible except in the gapless phase induced by a magnetic field, where they will open a gap. The intriguing properties of the famous plateau system $\mathrm{SrCu}_{2}\left(\mathrm{BO}_{3}\right)_{2}$ [7], in particular, the recent report that the translational symmetry remains broken above the $1 / 8$ plateau [8], suggest this might not, however, be so simple. Indeed, significant DM interactions have been identified in this system, and the role they may play between the plateaux remains to be investigated. In the same spirit, the nature of the phase transition of the intermediate phase of the spin-gap system $\mathrm{Cu}_{2}\left(\mathrm{C}_{5} \mathrm{H}_{12} \mathrm{~N}_{2}\right)_{2} \mathrm{Cl}_{4}$ [9] is still an open issue, and the recent identification of DM interactions in that system [10] opens new perspectives that remain to be explored.

In this Letter, we report on an extensive investigation of the effect of DM interactions in gapped systems in the context of spin ladders, and we show that even very small DM interactions can induce Ising phase transitions with broken translational symmetry in all spin ladders, frustrated or not, under appropriate conditions. To cover all aspects of the problem, we concentrate on a fairly general model depicted in Fig. 1. Its Hamiltonian can be split into single-rung exchange, DM, and Zeeman energies,

$$
\begin{aligned}
\mathcal{H}_{\text {rung }}= & J \sum_{j} \mathbf{S}_{j, 1} \cdot \mathbf{S}_{j, 2}+\sum_{j} \mathbf{D} \cdot\left(\mathbf{S}_{j, 1} \times \mathbf{S}_{j, 2}\right) \\
& -\sum_{j} \mathbf{H} \cdot\left(\mathbf{S}_{j, 1}+\mathbf{S}_{j, 2}\right)
\end{aligned}
$$

and Heisenberg interactions between adjacent rungs,

$$
\begin{aligned}
\mathcal{H}_{\text {int }}= & J_{\|} \sum_{j}\left(\mathbf{S}_{j, 1} \cdot \mathbf{S}_{j+1,1}+\mathbf{S}_{j, 2} \cdot \mathbf{S}_{j+1,2}\right) \\
& +J_{X} \sum_{j}\left(\mathbf{S}_{j, 1} \cdot \mathbf{S}_{j+1,2}+\mathbf{S}_{j, 2} \cdot \mathbf{S}_{j+1,1}\right) .
\end{aligned}
$$

In this work, the magnetic field $\mathbf{H}=(0,0, H)$ is applied perpendicular to the $\mathrm{DM}$ vector $\mathbf{D}=(0, D, 0)$, which is taken to be uniform, the relevant configuration for ladders that are translationally invariant but have no inversion center in the middles of the rungs. The full symmetry group of the system can be generated from three symmetry operations: $T \sigma_{y z}, C_{2}(z)$, and $T \sigma_{x z}$, as shown in Fig. 1, where the additional time-reversal operator $T$ is needed to compensate for the presence of the magnetic field. The symmetry operations act both in spin and real space.

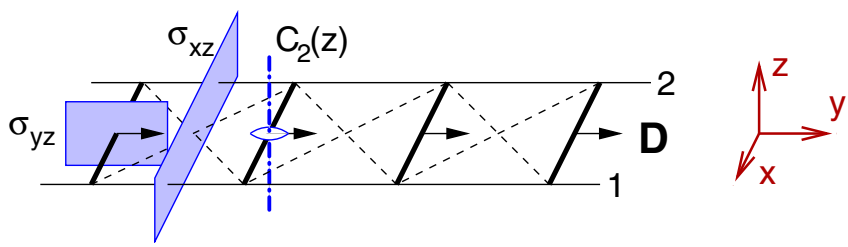

FIG. 1 (color online). Sketch of the frustrated ladder of Eqs. (1) and (2). The thick solid lines stand for $J$, the thin ones for $J_{\|}$, the dashed ones for $J_{X}$, and the arrows for $\mathbf{D}$. We have also shown the 3 generators of the space group: the $\sigma_{x z}$ and $\sigma_{y z}$ mirror planes and a twofold rotation axis $C_{2}(z)$. 
The physics of this model is controlled by two factors. (i) The level of frustration of the Heisenberg exchange. Indeed, when $D=0$, a magnetization plateau at half the saturation has been shown to open in strongly frustrated ladders $\left(1 / 3<J_{X} / J_{\|}<3\right)$, corresponding to a gapped phase with a broken translational symmetry [5]. By contrast, nonfrustrated ladders $\left(J_{X}=0\right.$ or $\left.J_{\|}=0\right)$ and weakly frustrated ladders $\left(0<J_{X} / J_{\|}<1 / 3\right.$ or $\left.0<J_{\|} / J_{X}<1 / 3\right)$ remain in a gapless Luttinger liquid phase from zero magnetization to full polarization. (ii) The possible competition between exchange and DM interactions [11]. In the present context, this can be understood as follows. Because of the DM interaction, an isolated rung in a field develops opposite moments along $\mathbf{D} \times \mathbf{H}$, on the two sites of the dimer [12]. If such dimers are coupled in a ladder geometry, an antiferromagnetic inter-rung coupling $J_{\|}$will compete with the DM interaction because $J_{\|}$tends to align the moments antiparallel along a leg. By contrast, an antiferromagnetic inter-rung coupling $J_{X}$ will not compete since both $J_{X}$ and the DM interactions induce antiparallel moments along the diagonals.

The noncompeting case $\left(J_{X} / J_{\|}>1\right)$ has been studied previously [13]. The DM interaction opens a gap in the gapless incommensurate phase, as expected, but only induces a small shift of the Ising plateau transition in strongly frustrated ladders. In the following, we will concentrate on the competing case $\left(J_{X} / J_{\|}<1\right)$.

The simplest way to map out the phase diagram is to use a Hartree variational function of the form

$$
\psi=\prod_{j}\left(\cos \frac{\theta_{j}}{2}|T\rangle+e^{i \varphi_{j}} \sin \frac{\theta_{j}}{2}|S\rangle\right),
$$

with $|T\rangle=\left|\uparrow_{1} \uparrow_{2}\right\rangle$ and $|S\rangle=\left(\left|\downarrow_{1} \uparrow_{2}\right\rangle-\left|\uparrow_{1} \downarrow_{2}\right\rangle\right) / \sqrt{2}$ on the $j$ th rung [14]. The trial state is characterized by the symmetric and antisymmetric magnetizations of a rung, $\mathbf{m}_{j \pm}=\left\langle\mathbf{S}_{j, 1} \pm \mathbf{S}_{j, 2}\right\rangle$. The nonzero components are

$$
m_{j,-}^{x}+i m_{j,-}^{y}=\sin \theta_{j} e^{i \varphi_{j}} / \sqrt{2}, \quad m_{j,+}^{z}=\cos ^{2}\left(\theta_{j} / 2\right) .
$$

The variational phase diagram without DM interaction is shown in Fig. 2(a). It is symmetric with respect to the exchange of $J_{X}$ and $J_{\|}$and contains all the phases found previously [13]: two fully symmetric integer plateau states with $m_{j,+}^{z}=0$ and 1; a half-integer plateau phase with a broken $Z_{2}$ translational symmetry, $m_{j,+}^{z}-1 / 2 \propto(-1)^{j}$, and a gapless phase with a spontaneously broken $\mathrm{O}(2)$ symmetry [the broken $\mathrm{O}(2)$ being an artifact of the mean-field approximation].

The variational phase diagram in the presence of the DM interaction is shown in Fig. 2(b). It is clearly related to the $\mathrm{O}(2)$-symmetric case: some of the transition lines lie close to their $D=0$ counterparts. However, there are some notable differences. In the noncompeting regime, $J_{X} / J_{\|}>$ 1 , the phase transitions between the integer plateaux and the intermediate gapless phase are replaced with crossovers, in agreement with previous work [13].
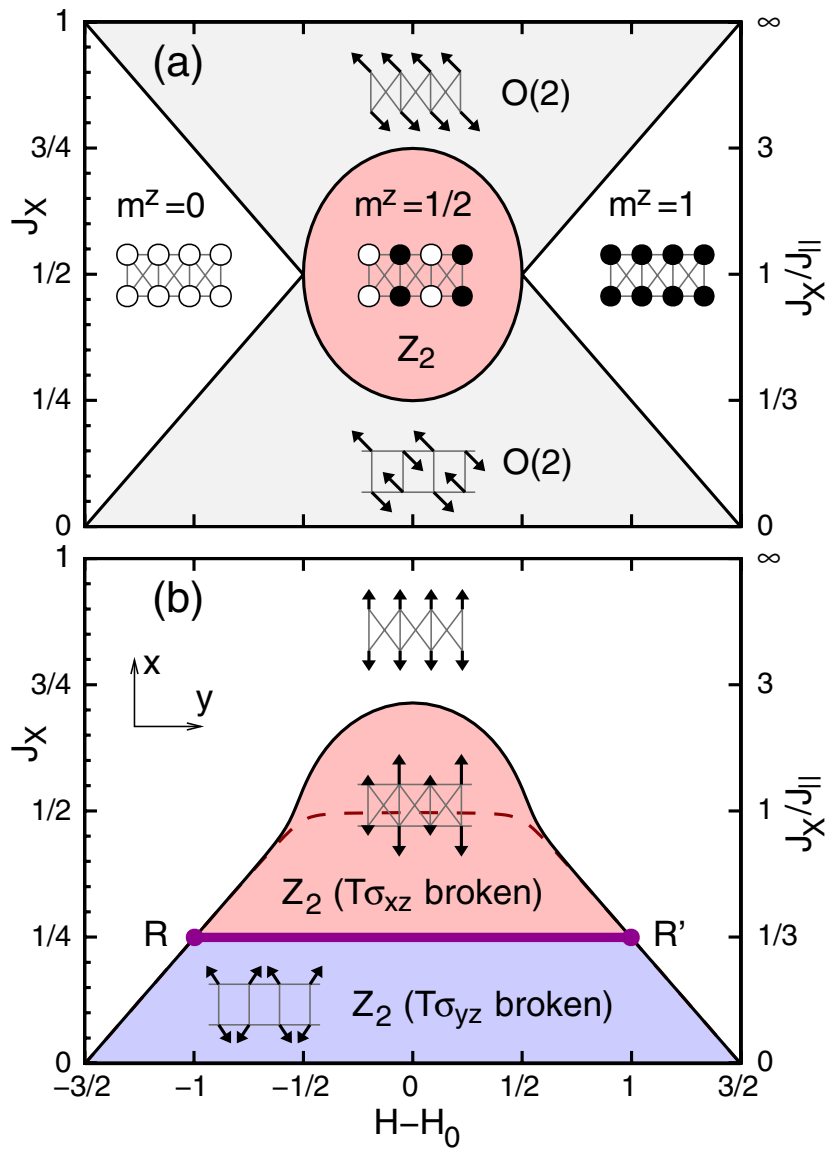

FIG. 2 (color online). Variational phase diagram for (a) $D=0$ and (b) $D /\left(J_{X}+J_{\|}\right)=0.1$. On the left and bottom axes, $J_{X}$ and $H-H_{0}$, where $H_{0}=J+\left(J_{X}+J_{\|}\right) / 2$, are given in the units of $J_{X}+J_{\|}$. In (a), the spins forming singlets and triplets in the gapped phase are represented by open and solid circles. In the gapless $O(2)$ phase the arrows show a typical mean-field ground state. In (b), the $O(2)$ symmetry is broken by finite $D$ : a unique state is selected in the nonfrustrated case, a twofold degeneracy is found in the frustrated case. The Ising phase of the plateau in (a) develops continuously by turning on $D$. Along the red dashed line the site-factorized wave function is an exact wave function in the large- $J$ limit. $R$ and $R^{\prime}$ denote the tricritical points.

Far more dramatic changes can be seen in the competing regime, $J_{X} / J_{\|}<1$. At strong frustration, $1 / 3<J_{X} / J_{\|}<$ 1 , the Ising-ordered phase has substantially expanded its boundaries by absorbing the incommensurate phases. A distinct Ising order appears for zero or weak frustration, $0 \leq J_{X} / J_{\|}<1 / 3$. In the upper Ising phase, the order parameter is the staggered component of the magnetizations $m_{j,+}^{z}$ and $m_{j,-}^{x}$. It breaks $T \sigma_{x z}$. In the lower Ising phase, the order parameter is the staggered component of $m_{j,-}^{y}$. It breaks $T \sigma_{y z}$. Since $T \sigma_{y z} C_{2}(z) T \sigma_{x z}$ is the translation operator, both phases break the translational invariance as well. In any of the two phases, only one generator of the space group is broken; thus, the transitions from the uniform phase into these phases are continuous. At the variational level, the transition between the two Ising 


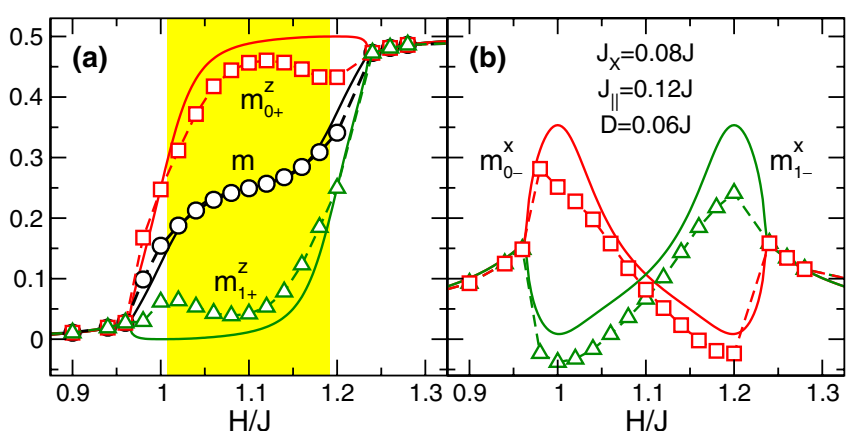

FIG. 3 (color online). (a) Uniform magnetizations of neighboring rungs $\left(m_{0+}^{z}\right.$ and $\left.m_{1+}^{z}\right)$ and average $(m)$ in the upper Ising phase and (b) staggered magnetizations $m_{0+}^{x}$ and $m_{1+}^{x}$. Solid lines: variational approach; symbols and dotted lines: DMRG for 58 sites; shaded area: plateau phase when $D=0$.

phases is first order, but numerical results and field-theory arguments suggest that the transition is made continuous by quantum critical fluctuations (see below).

To test the reliability of the variational approach, we have obtained the ground state and magnetization distributions for the ladder model (1) and (2) using the density matrix renormalization group (DMRG) for two representative cases: the unfrustrated ladder $\left(J_{X}=0\right)$ and a strongly frustrated one $\left(J_{X} / J_{\|}=2 / 3\right)$, and we have obtained strong evidence for the existence of the two Ising phases. The results for relevant local magnetizations are compared with the variational results in Figs. 3 and 4. In the upper Ising phase $\left(J_{X} / J_{\|}=2 / 3\right.$, Fig. 3$)$, the agreement is nearly perfect and the presence of a broken symmetry far outside the half-integer magnetization plateau is well established: magnetizations $m_{j,+}^{z}$ of two neighboring rungs exhibit a finite difference in almost the entire range of fields between the two integer magnetization plateaux. For $J_{X} / J_{\|}=0$ (see Fig. 4), we have included a small staggered field to allow the finite system to maintain a nonzero staggered magnetization along the $D$ vector. We find a qualitative agreement between the variational

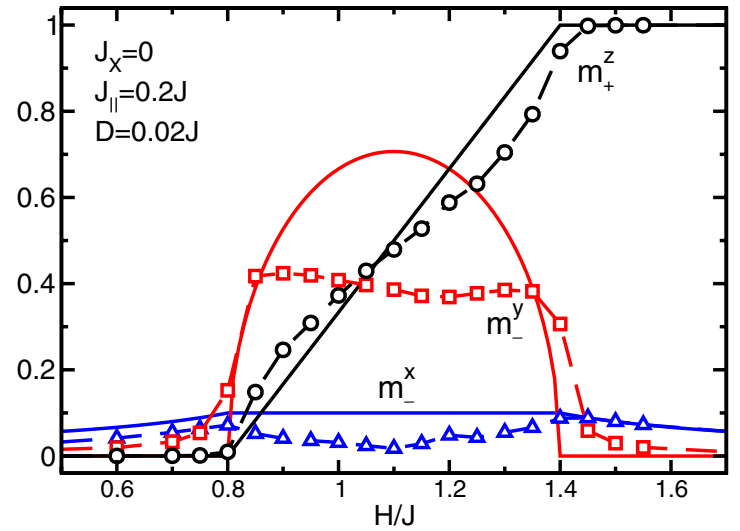

FIG. 4 (color online). Magnetizations in the lower Ising phase. Solid lines: variational approach; symbols and dotted lines: DMRG for 80 sites. and numerical results. Quantitatively, the mean-field approach overestimates the order parameter by as much as a factor of 2, presumably the result of neglecting quantum fluctuations.

To understand the physics of the ordered states and phase transitions between them we turn to the strongcoupling limit where the rung exchange $J$ of Eq. (1) dominates. The low-energy physics of the model then reduces to that of a spin-1/2 XXZ chain [5],

$$
\begin{aligned}
\mathcal{H}_{\mathrm{eff}}= & j \sum_{i}\left(\sigma_{i}^{x} \sigma_{i+1}^{x}+\sigma_{i}^{y} \sigma_{i+1}^{y}+\Delta \sigma_{i}^{z} \sigma_{i+1}^{z}\right) \\
& -\sum_{i}\left(h_{x} \sigma_{i}^{x}+h_{z} \sigma_{i}^{z}\right),
\end{aligned}
$$

with $j=J_{\|}-J_{X}$ and $\Delta=\left(J_{X}+J_{\|}\right) / 2\left(J_{\|}-J_{X}\right)$. The longitudinal field is related to the field in the original problem, $h_{z}=H-J-\left(J_{X}+J_{\|}\right) / 2$, while the transverse field comes from the DM interactions, $h_{x}=D / \sqrt{2}$, as discussed previously [15]. The spin- $1 / 2$ operators $\sigma$ are related to the original spin operators in the following way:

$$
\sigma_{j}^{\alpha}=P \frac{S_{j, 1}^{\alpha}-S_{j, 2}^{\alpha}}{\sqrt{2}} P, \quad \sigma_{j}^{z}=P\left(S_{j, 1}^{z}+S_{j, 2}^{z}-\frac{1}{2}\right) P,
$$

where $\alpha=x, y$ and $P=\left(S_{j, 1}^{+}-S_{j, 2}^{+}\right)\left(S_{j, 1}^{-}-S_{j, 2}^{-}\right) / 2$ is the projector onto the subspace $\{|S\rangle,|T\rangle\}$. Nonzero averages $\left\langle\sigma_{j}^{x}\right\rangle$ and $\left\langle\sigma_{j}^{y}\right\rangle$ signal the appearance of antisymmetric magnetizations $m_{j,-}^{x}$ and $m_{j,-}^{y}$, while $\left\langle\sigma^{z}\right\rangle$ translates into the symmetric magnetization $m_{j,+}^{z}-1 / 2$; cf. Eq. (4).

The $X X Z$ model in a tilted uniform field (5) has been studied previously [16,17]. The magnetic order is lost across the line of Ising transitions, $h_{z} \approx(\Delta+1) j$, into the fully magnetized plateaux. The two Ising phases are separated by the Heisenberg line $\Delta=1$ along which the system has an axial symmetry. For $h=|\mathbf{h}|<2 j$, the system is gapless and can be viewed as a Luttinger liquid of magnons, whose dimensionless compressibility $K$ [4] varies between 1 (a dilute magnon gas near the fully magnetized state) and $1 / 2$ (a dense magnon fluid at low magnetization) [18].

To discuss the physics away from the Heisenberg point, it is convenient to make a global rotation in the $x z$ plane, defining a new quantization axis $z^{\prime}$ aligned with the total field $\mathbf{h}$. When $\Delta \neq 1$, this rotation generates new terms in the Hamiltonian. The nature of the phase transitions becomes particularly transparent in the vicinity of the tricritical points $R$ and $R^{\prime}$ located at $\Delta=1, h= \pm 2 j$, where the system can be described as a dilute magnon gas. Then the main effect is the lowering of the axial symmetry by a weak anisotropy induced in the $x^{\prime} y$ plane,

$$
\lambda \sum_{j}\left(\sigma_{j}^{x^{\prime}} \sigma_{j+1}^{x^{\prime}}-\sigma_{j}^{y} \sigma_{j+1}^{y}\right)=\frac{\lambda}{2} \sum_{j}\left(\sigma_{j}^{+} \sigma_{j+1}^{+}+\sigma_{j}^{-} \sigma_{j+1}^{-}\right),
$$

where $\lambda \sim(\Delta-1) h_{x}^{2} / 8 j$. The magnon Luttinger liquid becomes gapped by developing a staggered magnetization 


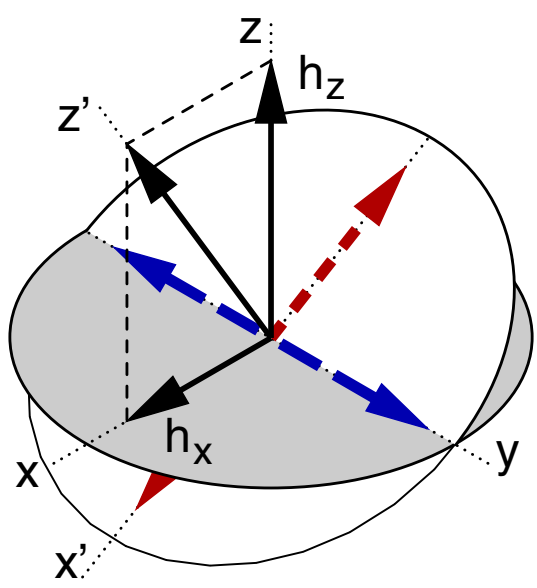

FIG. 5 (color online). Schematic representation of the phase transitions. Away from the $\Delta=1$ Heisenberg line, the $\mathcal{H}_{\text {eff }}$ loses the axial symmetry around the total field: the spins develop staggered order along the long dashed blue arrows if $\Delta<1$ and along the short dashed red arrows if $\Delta>1$.

along $y$ if $\Delta<1$ (breaking $T \sigma_{x y}$ [19]) or along $x^{\prime}$ if $\Delta>1$ (breaking $T \sigma_{x z}$ [19]), see Fig. 5. The anisotropy (6) represents a pairing field for the magnons. From the scaling dimension of the pairing operator we infer that the energy gap in the magnon spectrum should scale as $E_{g} \propto|\lambda|^{\nu}$ with a nonuniversal exponent $\nu=K /(2 K-1)$.

The global rotation also generates a 3-body term [16],

$$
\frac{(\Delta-1) h_{x}}{4} \sum_{j}\left(\sigma_{j}^{+}+\sigma_{j}^{-}\right)\left(\sigma_{j-1}^{-} \sigma_{j-1}^{+}+\sigma_{j+1}^{-} \sigma_{j+1}^{+}\right) .
$$

which has only a minor effect on the physics near the critical line separating the Ising phases. While low-energy magnons live near lattice momentum $\pi$, this term creates 1- and 3-magnon states with a total momentum 0 , which are thus high-energy excitations. Therefore the 3-body term does not affect the low-energy physics to the first order. In the second order it generates 2- and 4-magnon terms that simply renormalize the magnon velocity, compressibility $K$, and pairing field $\lambda$ but do not change the physics qualitatively. The existence of two distinct ordered phases is confirmed by an exact solution on a special line in the phase diagram where the mean-field approach yields the correct result [16]. The Heisenberg line $\Delta=1$ is guaranteed to be critical by the $\mathrm{O}(2)$ symmetry.

To summarize, we have shown that even a weak DM interaction can substantially alter the phase diagram of ladders, provided that it competes with exchange. For strongly frustrated ladders, the phase with a broken translational symmetry expands to include the incommensurate regions surrounding the fractional plateau, while for unfrustrated and weakly frustrated ladders, the gapless phase undergoes an Ising transition that also breaks translation, but with a different symmetry. Beyond ladders, we expect to find similar effects in higher dimensional models of coupled spin-1/2 dimers, whenever DM and exchange compete. In fact, the broken translational symmetry above the 1/8 plateau of $\mathrm{SrCu}_{2}\left(\mathrm{BO}_{3}\right)_{2}$ might be an example of the frustrated case, and the ordered intermediate phase of $\mathrm{Cu}_{2}\left(\mathrm{C}_{5} \mathrm{H}_{12} \mathrm{~N}_{2}\right)_{2} \mathrm{Cl}_{4}$ of the unfrustrated one. However, taking into account the specific (and complex) geometries of these systems is necessary to go beyond these qualitative observations.

We acknowledge enligthening discussions with C. Berthier, M. Horvatic, S. Matsubara, B. Normand, and M. Takigawa. This work was supported by the Hungarian OTKA Grants No. T049607 and No. K62280, by the Swiss National Fund, by MaNEP, and by the U.S. National Science Foundation Grant No. DMR-0348679.

[1] E. Dagotto and T. M. Rice, Science 271, 618 (1996).

[2] E. Dagotto, Rep. Prog. Phys. 62, 1525 (1999).

[3] B. C. Watson et al., Phys. Rev. Lett. 86, 5168 (2001).

[4] T. Giamarchi, Quantum Physics in one Dimension (Oxford University, Oxford, 2004).

[5] F. Mila, Eur. Phys. J. B 6, 201 (1998).

[6] M. Oshikawa, M. Yamanaka, and I. Affleck, Phys. Rev. Lett. 78, 1984 (1997).

[7] S. Miyahara and K. Ueda, J. Phys. Condens. Matter 15, R327 (2003).

[8] M. Takigawa, K. Kodama, M. Horvatic, C. Berthier, S. Matsubara, H. Kageyama, Y. Ueda, S. Miyahara, and F. Mila, J. Phys.: Conf. Ser. 51, 23 (2006).

[9] G. Chaboussant, M.-H. Julien, Y. Fagot-Revurat, M. Hanson, L. P. Levy, C. Berthier, M. Horvatic, and O. Piovesana, Eur. Phys. J. B 6, 167 (1998).

[10] M. Clémancey, H. Mayaffre, C. Berthier, M. Horvatić, J.-B. Fouet, S. Miyahara, F. Mila, B. Chiari, and O. Piovesana, Phys. Rev. Lett. 97, 167204 (2006).

[11] M. Sato and M. Oshikawa, Phys. Rev. B 69, 054406 (2004).

[12] S. Miyahara, J.-B. Fouet, S. R. Manmana, R. M. Noack, H. Mayaffre, I. Sheikin, C. Berthier, and F. Mila, Phys. Rev. B 75, 184402 (2007).

[13] J.-B. Fouet, F. Mila, D. Clarke, H. Youk, O. Tchernyshyov, P. Fendley, and R. M. Noack, Phys. Rev. B 73, 214405 (2006). Note that in this reference, the DM vector is taken to alternate from one rung to the other, so that the weakly frustrated regime corresponds to weak diagonal interactions.

[14] T. Momoi and K. Totsuka, Phys. Rev. B 62, 15067 (2000).

[15] M. Oshikawa and I. Affleck, Phys. Rev. B 65, 134410 (2002).

[16] A. A. Ovchinnikov, D. V. Dmitriev, V. Y. Krivnov, and V. O. Cheranovskii, Phys. Rev. B 68, 214406 (2003); D. V. Dmitriev and V. Y. Krivnov, Phys. Rev. B 70, 144414 (2004).

[17] J.-S. Caux, F. H. L. Essler, and U. Löw, Phys. Rev. B 68, 134431 (2003).

[18] F. D. M. Haldane, Phys. Rev. Lett. 45, 1358 (1980).

[19] The symmetry generators $C_{2}(z), T \sigma_{y z}$ and $T \sigma_{x z}$ of the ladder transform $\left(\sigma_{j}^{x^{\prime}, z^{\prime}}, \sigma_{j}^{y}\right)$ into $\left(\sigma_{-j}^{x^{\prime}, z^{\prime}}, \sigma_{-j}^{y}\right),\left(\sigma_{j}^{x^{\prime}, z^{\prime}},-\sigma_{j}^{y}\right)$ and $\left(\sigma_{1-j}^{x^{\prime}, z^{\prime}},-\sigma_{1-j}^{y}\right)$, respectively. 